\title{
Au/Ag-containing DNA-based nanowires
}

\author{
Dmitry Basmanov ${ }^{1, *}$, Kirill Prusakov ${ }^{1,2}$, Dmitry Klinov ${ }^{1}$, Gennady Eidelstein ${ }^{3}$, \\ and Aleksander Kotlyar ${ }^{3}$ \\ ${ }^{1}$ Federal State Budgetary Institution Federal Research and Clinical Center Of Physical-Chemical \\ Medicine Federal Medical Biological Agency, Laboratory of Medical Nanotechnologies, 119435 \\ Malaya Pirogovskaya 1a, Moscow, Russia \\ ${ }^{2}$ Moscow institute of physics and technology, Departure of Biological and Medical Physics, 141701 \\ Institutskiy per. 9, Dolgoprudny, Moscow Region, Russia \\ ${ }^{3}$ Department of Biochemistry and Molecular Biology, Faculty of Life Sciences and Center of \\ Nanoscience and Nanotechnology, Tel Aviv University, Ramat Aviv, Tel Aviv 69978, Israel
}

\begin{abstract}
Direct conductivity measurements of thin uniform gold-coated DNA nanowires are presented. Gold-coated DNA conductive wires deposited on a mica surface are visualized by SEM and conductivity of the wires are measured using nanomanipulators built inside the microscope. The measurements show that the conductivity is limited by defects, and the thicker the coating the higher the conductivity of the wires. These goldcoated DNA nanowires are promising candidates for molecular electronics. DNA-base nanomaterial to be used in new DNA-based molecular electronics.
\end{abstract}

\section{Introduction}

DNA is considered to be one of the most promising candidates for future molecular electronics. Several types of DNA-based metalized nanowires have been recently reported. Among them are: new metalized form of DNA, E-DNA [1], and thin gold nanoparticlescoated DNA wires [2]. In this work we present direct conductivity measurements of such gold-coated DNA wires.

\section{Materials and Methods}

The suspension of gold seeds (diameter of about $1.5 \mathrm{~nm}$ ) was poured on a mica substrate with deposited DNA and left on the surface for $4 \mathrm{~h}$. The surface was then washed with ultrapure distilled water and dried by a nitrogen flow. The mica surface was subsequently flowed with a freshly premixed enhancement solutions of ascorbic acid. The ascorbic acid and the gold solutions were inserted into a mixing chamber by two peristaltic pumps, such that the mixture flushed the surface $\approx 1 \mathrm{~s}$ after the mixing. Treatment of the surface with the mixture was conducted for $10 \mathrm{~s}$. The surface was then rinsed with water and dried with nitrogen gas [2].

\footnotetext{
* Corresponding author: dmitry.basmanov@niifhm.ru
} 
This samples were visualized by Zeiss Merlin with GEMINI II Electron Optics SEM. The measurements of gold-coated DNA wires conductivity were performed with Kleindiek nanotechnik MM3A-EM micromanipulators mounted on an SEM adaptor plate inside the SEM vacuum chamber, and Keithley 2636A System SourceMeter (see Fig. 1).

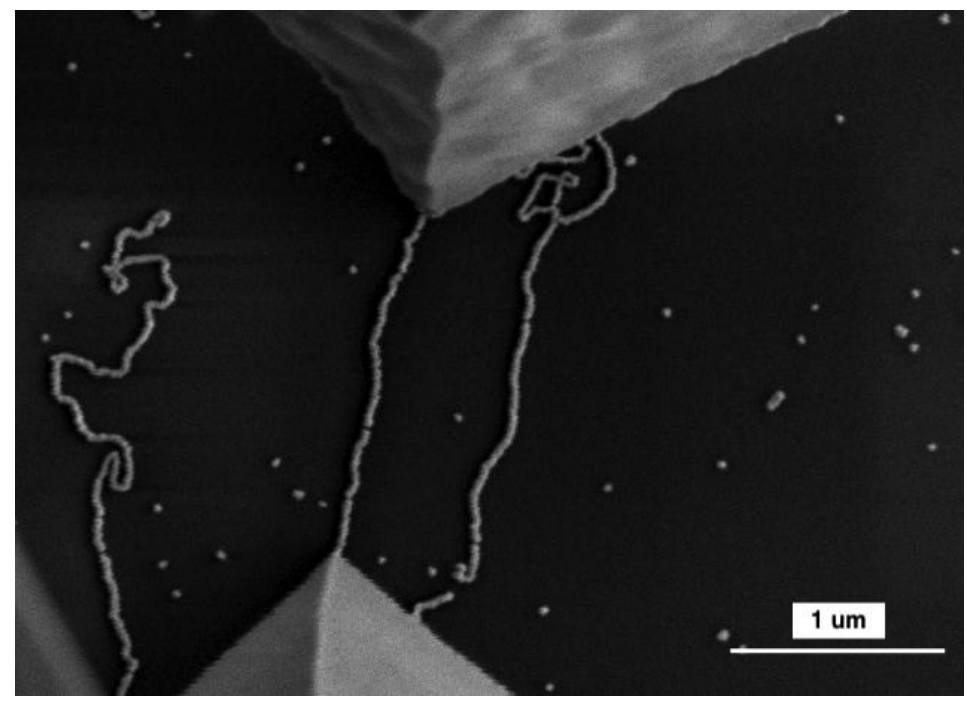

Fig. 1. Measurements of VAC of gold-coated DNA wires inside the Zeiss Merlin SEM.

\section{Results and Conclusion}

The thin gold-coated DNA wires display long-range conductivity. Our measurements show that the conductivity is limited by defects. Moreover, the thicker the gold coating the less the number of defects and the longer the conduction distance of the wire. The preparation method enables the formation of molecular wires with dimensions and uniformity that are suitable for molecular electronics.

This work was supported by the Russian Science Foundation (project № 17-75-30064).

\section{References}

1. G. Eidelshtein, N. Fardian-Melamed, V. Gutkin, D. Basmanov, D. Klinov, D.Rotem, Y. Levi-Kalisman, D. Porath, and A. Kotlyar, Adv. Mater, 28, 4839 (2016)

2. A. Stern, G. Eidelshtein, R. Zhuravel, G. I. Livshits, D. Rotem, A. Kotlyar and D. Porath, Adv. Mater, 30, 1800433 (2018) 